\title{
ON CLOSED CURVES IN MINKOWSKI SPACES
}

\author{
H. S. WITSENHAUSEN
}

Abstracr. The minimum pseudo-diameter $d$ and the length $L$ of a simple closed rectifiable curve in Minkowski space satisfy $L \geqq g d$ where $g$ is the half-girth of the unit ball. The bound is sharp.

This note answers some of the questions raised by $\mathrm{H}$. Herda in [1].

Let $M$ be a Minkowski (finite dimensional real Banach) space with unit ball $B$. Let $\gamma$ be a simple closed rectifiable curve in $M$. For $x$ on $\gamma$ let $x^{\prime}$ be the point whose distance along $\gamma$ to $x$ is half the length $L(\gamma)$ of the curve $\gamma$. Let $d(\gamma)=\min _{x \in \gamma}\left\|x-x^{\prime}\right\|$ and let $g$ be the half-girth [2] of $B$, that is, the shortest length of a curve on $\partial B$ with antipodal endpoints.

THEOREM 1. One has $L(\gamma) \geqq g d(\gamma)$ for all $\gamma$ and there exists a curve for which equality holds.

Proof. Let $\gamma$ be given. Choose a point $a_{1}$ and an orientation on $\gamma$. For arbitrary $\varepsilon \in\left(0, \frac{1}{2}\right)$ choose an integer $k>0$ such that $2^{-k} L(\gamma)<\varepsilon d(\gamma)$. Define points $a_{i} \in \hat{\gamma}, i=1, \cdots, 2^{k}$, succeeding each other in the chosen direction with the $\gamma^{\prime}$-arc from $a_{i}$ to $a_{i+1}$ of length $2^{-k} L(\gamma)$. Then $a_{i}^{\prime}=a_{j}$ where $j \equiv i+2^{k-1} \bmod 2^{k}$. Join the $a_{i}$ by straight line segments in index order to form a closed polygon $\alpha$. Let $r_{i}=\frac{1}{2}\left(a_{i}-a_{i}^{\prime}\right), m_{i}=\frac{1}{2}\left(a_{i}+a_{i}^{\prime}\right)$.

Join the points $r_{i}$ in index order to form a closed polygc $n$, centrally symmetric about the origin. By convexity of the norm one has

$$
\begin{aligned}
\left\|a_{i+1}-a_{i}\right\|+\left\|a_{i \cdot 1}^{\prime}-a_{i}^{\prime}\right\|= & \left\|\left(m_{i \cdot 1}-m_{i}\right)+\left(r_{i+1}-r_{i}\right)\right\| \\
& +\left\|\left(m_{i+1}-m_{i}\right)-\left(r_{i \uparrow 1}-r_{i}\right)\right\| \\
\geqq & 2\left\|r_{i+1}-r_{i}\right\| .
\end{aligned}
$$

Since $\left\|a_{i+1}-a_{i}\right\| \leqq 2^{-k} L(\gamma)$ for all $i$, one has

$$
\left\|r_{i: 1}-r_{i}\right\| \leqq 2^{-k} L(\gamma)<\varepsilon d(\gamma) .
$$

By construction $\left\|r_{i}\right\|=\frac{1}{2}\left\|a_{i}-a_{i}^{\prime}\right\| \geqq \frac{1}{2} d(\gamma)$. All vertices of polygon $\rho$ are therefore on the boundary or exterior of the ball $\frac{1}{2} d\left(\gamma^{\prime}\right) B$. Then no point

Received by the editors November 24, 1971.

AMS 1970 subject classifications. Primary 52A40; Secondary 50C25, 46B99.

Key words and phrase's. Minkowski spaces, pseudo-diameters, girth of balls. 
of $\rho$ can lie in the interior of the ball $\left(\frac{1}{2}-\varepsilon\right) d(\gamma) B$. Therefore

$$
\begin{aligned}
L(\gamma) & \geqq L(\alpha)=\sum_{i=1}^{2^{k-1}}\left(\left\|a_{i+1}-a_{i}\right\|+\left\|a_{i+1}^{\prime}-a_{i}^{\prime}\right\|\right) \\
& \geqq 2 \sum_{i=1}^{2^{k-1}}\left\|r_{i+1}-r_{i}\right\|=L(\rho) \geqq(1-2 \varepsilon) g d(\gamma)
\end{aligned}
$$

where $g$ is the half-girth of $B$, the last inequality by Lemmas 3.2 and 5.1 (a) of Schäffer [2]. This proves that the inequality holds. By Lemma 5.1(b) of [2] there exists a curve $\gamma$ on $\partial B$, centrally symmetric about the origin, for which $L(\gamma)=2 g$ and, trivially, $d(\gamma)=2$, yielding equality.

REMARKS. 1. In infinite dimension the same argument applies but a curve yielding equality may not exist.

2. In 2 dimensions, $L(\gamma)=g d(\gamma)$ does not imply that $\gamma$ is a homothet of $\partial B$. When the unit ball is a square, an infinity of curves exists which have $L(\gamma)=8, d(\gamma)=2$ and up to twice the area of $B$.

3. For Euclidean and Hilbert spaces R. Ault has shown [4] that $L=\pi d$ only for circles, settling Herda's conjecture in the affirmative. An earlier partial proof for $E^{2}$ was provided by A. M. Fink [3].

4. Herda's second conjecture states that for a simple closed rectifiable curve $\gamma$ in $E^{2}$ the following are equivalent:

(i) the curve admits a unique tangent at each point;

(ii)

$$
\inf _{t \in(0, d)} \max _{x \in \gamma} a(x, t) / t=1
$$

where $a(x, t)$ is the shortest arc length from point $x$, in a fixed orientation along $\gamma$, to a point of $\gamma$ at Euclidean distance $t$ from $x$. This conjecture is false. Let $\gamma_{1}$ be the graph in $E^{2}$ of the function $f$ defined on $[-1,+1]$ by $f(0)=0, f(x)=x^{2} \sin x^{-1}$. Complete $\gamma_{1}$ into a simple closed curve $\gamma$ by a smooth connection of its endpoints. Then $\gamma$ is rectifiable and has a unique tangent at each point while (ii) fails because

$$
\lim _{\varepsilon \rightarrow 0} \frac{1}{\varepsilon} \int_{0}^{\varepsilon}\left(1+\cos ^{2} t^{-1}\right)^{1 / 2} d t>1
$$

\section{REFERENCES}

1. H. Herda, A conjectured characterization of circles, Amer. Math. Monthly 78 (1971), 888-889.

2. J. J. Schäffer, Inner diameter, perimeter, and girth of spincres, Math. Ann. 173 (1967), 59-79; addendum, ibid. 173 (1967), 79-82. MR 36 \#1959.

3. A. M. Fink, A circle maximizes the minimum pscudo-diameter (written communication).

4. R. Ault, Metric characterization of circles (written communication).

Mathematics Research Center, Bell. Telephoni. Laboralorifs, Incorporated, Murray Hill, New Jersey 07974 